\title{
Efficacy of Methylprednisolone Injected into Masseter Muscle Preoperatively in Surgical Extraction of Lower Third Molars: A Randomized Placebo Controlled Triple Blind Studys
}

\author{
Diljith Rishi, Akshay Shetty*, Nikhil Srivastava, Rajani BC, Pratyush Anshuman and Pragati Kakkar
}

Faculty in Rajiv Gandhi Dental College, Oral And MaxilloFacial Surgeon, Dentist, Bangalore, India

\begin{abstract}
Background and objective: The administration of corticosteroids effectively reduces trismus, pain and facial swelling, but review of the literature has revealed few reports of administration of these drugs in the region adjacent to surgical trauma. The aim of this study is to evaluate the efficacy of methylprednisolone injected into the surgical site to reduce pain, swelling and trismus following the surgical extraction of impacted lower third molars and to develop protocols for effective use of corticosteroids in oral and maxillofacial surgery.
\end{abstract}

Methods: This randomized placebo controlled triple blind study was carried out between November 2012 to May 2014 on 15 patients requiring surgical removal of bilateral mandibular third molars. Patients were randomly allocated into the corticoid and control groups. The patient, operator and assessors were blinded with regards to the side of use of methylprednisolone $40 \mathrm{mg}$ and a placebo (injection saline) thus making it a triple blind study.

Results: In 13 out of $15(86.6 \%)$ patients the methylprednisolone group had better outcome in terms of less pain. In 12 out of $15(80 \%)$ patients the methylprednisolone group had better outcome in terms of less swelling and 11 out of $15(73.3 \%)$ patients the methylprednisolone group had better outcome in relation to trismus.

Interpretation and Conclusion: This randomized control trial has helped us to conclude that methylprednisolone definitely has a positive outcome in terms of reducing post-operative pain, swelling and trismus after lower third molar surgery.

Keywords: Methylprednisolone; Third molar; Intramassetric; Randomized control trial; Triple blind study

\section{Introduction}

Third molar extraction is one of the most common procedures performed in oral and maxillofacial surgery units. Patients complain about the pain, swelling and limitation in mouth opening associated with the inflammatory response following third molar surgical extractions as the factors affecting their daily life. Efforts to limit intraoperative or postoperative complications may have a great impact in terms of enhancing patient outcome [1]. Patients complain about the pain, swelling and limitation in mouth opening associated with the inflammatory response following third molar surgical extractions as the factors affecting their daily life [2]. This fact has led to the extensive use of a variety of medications to control these postoperative complications [3].

The incidence of excessive pain, swelling and trismus is reported as $12.3 \%, 8.6 \%$ and $5.7 \%$ respectively. Pain has been correlated to surgical extractions, suturing, bony impactions and the duration of surgery. Pain following third molar removal has in fact been used as a useful clinical model for the evaluation of analgesics. Swelling is correlated to surgical extractions, reflection of the mucoperiosteum and the duration of surgery. Trismus is correlated to surgical extractions, the duration of extraction and tooth sectioning. Swelling and trismus have been shown to be reduced with the use of glucocorticosteroids (local and systemic),nonsteroidal anti-inflammatory agents and systemic antibiotics, although the risk benefit ratio when using systemic antibiot $\neg$ ics does not justify their use for the reduction of swelling and trismus on a routine basis. Pain is influenced favourably by the perioperative administration of glucocorticosteroids and non-steroidal anti-inflammatory agents but is unaffected by the perioperative administration of systemic antibiotics [4].

Corticosteroids are potent inhibitors of inflammation, and they have been widely used in different routes and regimens to lessen the inflammatory sequelae after third molar surgery. Various corticosteroids such as betamethasone, triamcinolone, prednisolone, hydrocortisone, dexamethasone and methylprednisolone have been used to control pain, swelling and trismus postoperatively [5].

The selection of an appropriate glucocorticosteroid with minimal mineralocorticoid activity and extended biological activity is desirable.5Methylprednisolone meets these requirements, since it has no mineralocorticoid activity, the half-life is approximately 18-36 hours and the drug is 5 -fold more potent than hydrocortisone. There is a dearth of literature on the role of methylprednisolone for the postoperative complications of third molar surgery. So we decided to use the corticosteroid methylprednisolone (Figures 1-3).

It is widely accepted that peak corticosteroid levels are obtained by injecting the drug prior to the procedure. There was a need to conduct a randomized control trial which seeks to evaluate the efficacy of methylprednisolone injected into the masseter muscle via the intrabuccal approach prior to the procedure. Our study had twin objectives. One of them was to evaluate the efficacy of methylprednisolone injected into the surgical site to reduce pain, swelling and trismus following

*Corresponding author: Akshay Shetty, BDS, MDS, Faculty in Rajiv Gandhi Dental College, Bangalore, India, Tel: 0802354 6948; E-mail: akshayshetty7978@gmail.com

Received March 08, 2018; Accepted March 28, 2018; Published April 04, 2018

Citation: Rishi D, Rishi D, Shetty A, Srivastava N, Rajani BC, Anshuman P, et al., Srivastava N, Rajani BC, Anshuman P, et al. (2018) Efficacy of Methylprednisolone Injected into Masseter Muscle Preoperatively in Surgical Extraction of Lower Third Molars: A Randomized Placebo Controlled Triple Blind Study. Dentistry 8: 478. doi:10.4172/2161-1122.1000478

Copyright: (C) 2018 Rishi D, et al. This is an open-access article distributed unde the terms of the Creative Commons Attribution License, which permits unrestricted use, distribution, and reproduction in any medium, provided the original author and source are credited. 
Citation: Rishi D, Shetty A, Srivastava N, Rajani BC, Anshuman P, et al. (2018) Efficacy of Methylprednisolone Injected into Masseter Muscle Preoperatively in Surgical Extraction of Lower Third Molars: A Randomized Placebo Controlled Triple Blind Study. Dentistry 8: 478. doi:10.4172/2161-1122.1000478

Page 2 of 6
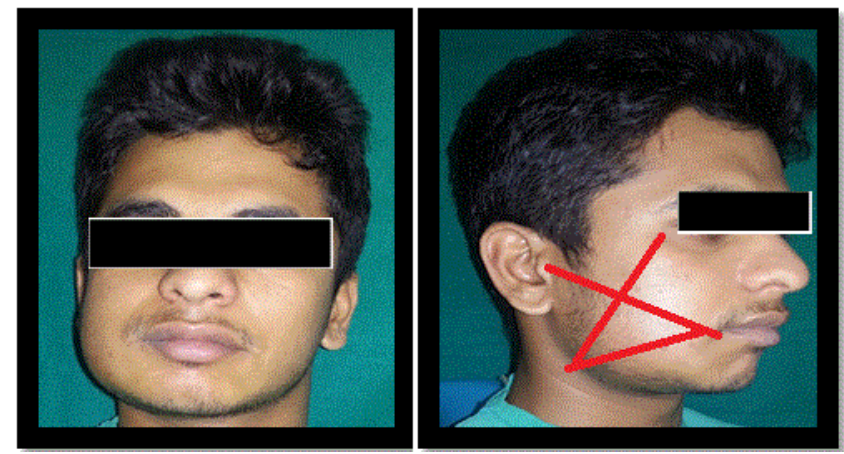

Figure 1: Markings for the measurement of facial swelling.

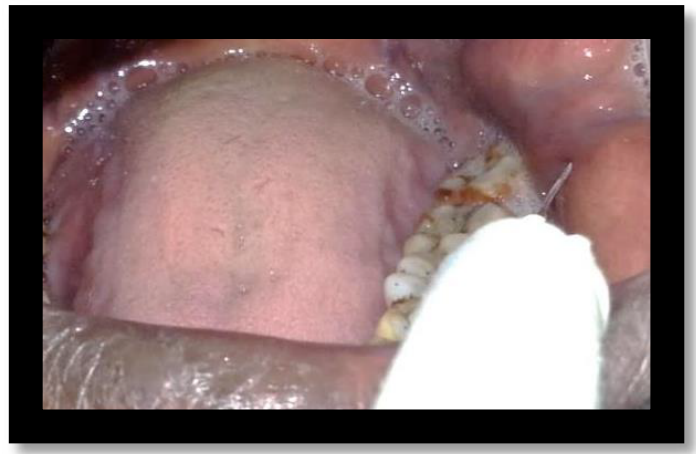

Figure 2: Intramassetric injection of methyprednisolone/placebo.

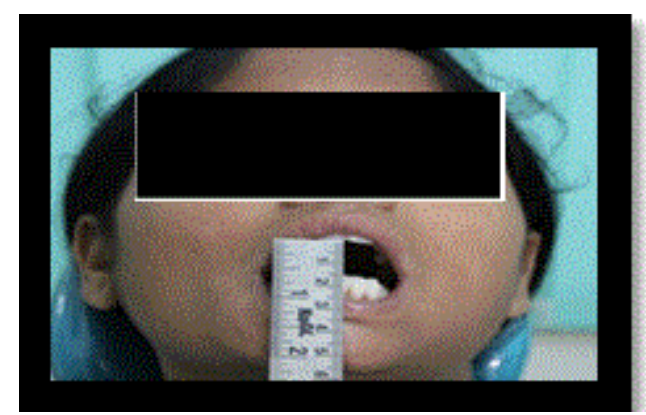

Figure 3: Measurement of trismus post-operative.

the surgical extraction of impacted lower third molars. The second objective was to develop protocols for effective use of corticosteroids in oral and maxillofacial surgery (Figure 4).

\section{Methodology}

This study was carried out on 15 patients who reported to the department of Oral and Maxillofacial surgery, Sri Rajiv Gandhi College of Dental Sciences\& Hospital Bangalore, requiring surgical removal of bilateral mandibular third molars.

\section{Inclusion criteria}

1. Patients who are not compromised systemically (ASA I).
2. Patients aged between 18 to 35 of either gender.

3. Patients requiring extraction of both impacted lower third molars.

4. Patients with identical level of difficulty of impacted lower third molars.

5. (Variation in Pedersen difficulty index of up to 2).

\section{Exclusion criteria}

6. Patients taking rescue drug within 6 hours postoperatively.

7. Patient not willing to be part of the study.6.3. Wax-up follows the smile frame exactly.

\section{Sample size estimation}

A sample size of 30 achieves $93 \%$ power to detect a mean of paired differences of 4.5 with an estimated standard deviation of differences of 3.2 and with a significance level (alpha) of 0.05000 using a two-sided paired t-test.

\section{Method of collection of data}

Patients were enrolled for the study consecutively as and when they reported to the department. Only those patients who met the inclusion and exclusion criteria were taken into account. All the patients were informed with regards to the purpose of the study and effects of the drug used. After taking informed consent of the patient and case history, all relevant findings were recorded using the proforma.

The patient, operator and assessors were blinded with regards to

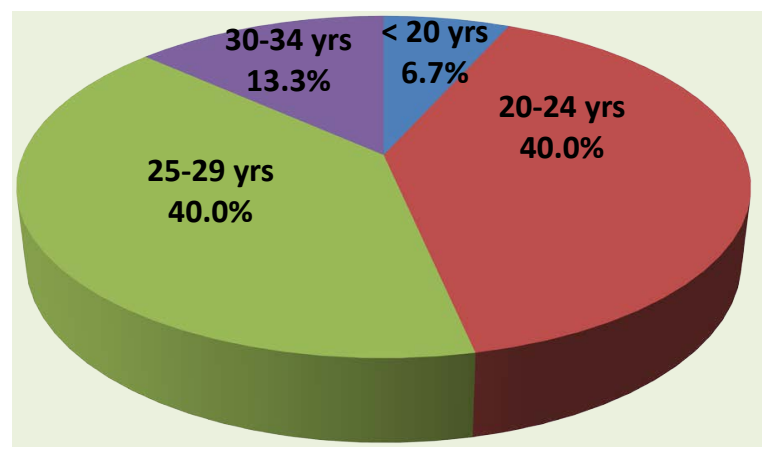

Figure 4: Age distribution of the Study Population $(\mathrm{N}=15)$.

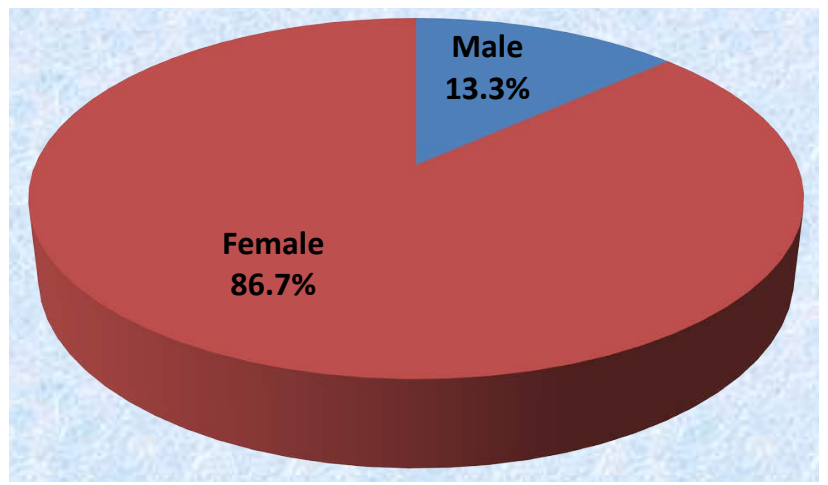

Figure 5: Gender distribution of the Study Population (N=15). 
Citation: Rishi D, Shetty A, Srivastava N, Rajani BC, Anshuman P, et al. (2018) Efficacy of Methylprednisolone Injected into Masseter Muscle Preoperatively in Surgical Extraction of Lower Third Molars: A Randomized Placebo Controlled Triple Blind Study. Dentistry 8: 478. doi:10.4172/2161-1122.1000478

Page 3 of 6

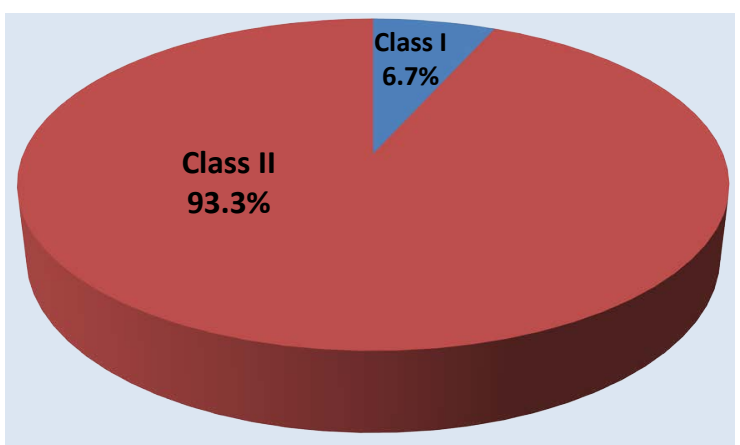

Figure 6: Distribution of Class according to Pell and Gregory Classification among the Study Population ( $\mathrm{N}=15)$.

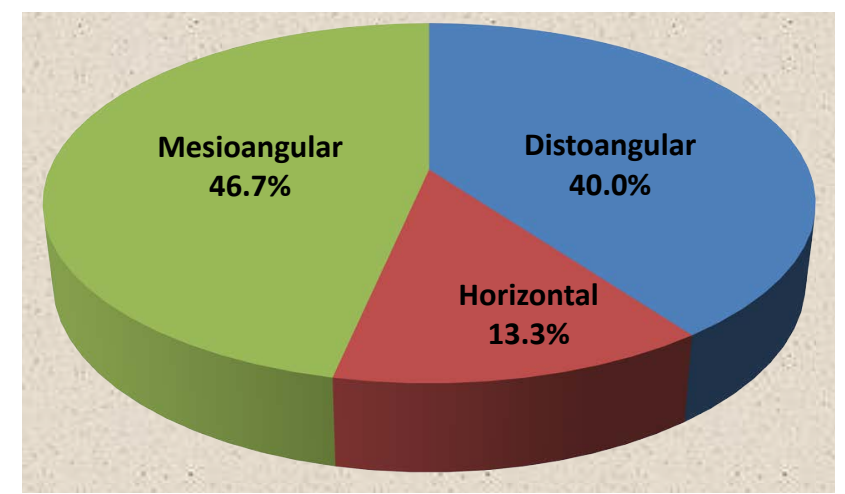

Figure 7: Distribution of Type of Impaction among the Study Population $(\mathrm{N}=15)$.

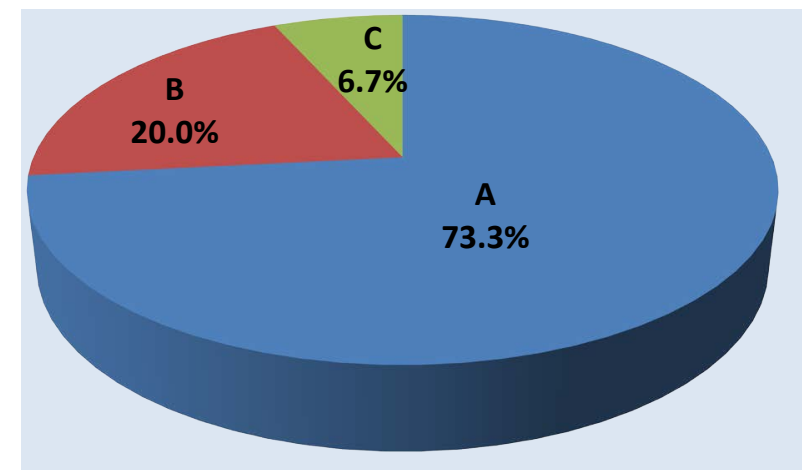

Figure 8: Distribution of Position accoding to Pell and Gregory Classification among the Study Population ( $\mathrm{N}=15)$.

the side of use of methylprednisolone $40 \mathrm{mg}$ and a placebo (injection saline) thus making it a triple blind study (Figures 5-8).

One of the MDS staff in the department injected the drug or the placebo. The syringe was taped with white plaster.

A chlorhexidine mouth rinse was given to the patient prior to starting the surgery.

- Patient was prepped and draped for surgery.

- Local Anesthesia was administered (2\% Lignocaine with adrenaline).
- Injection of methylprednisolone or the placebo (normal saline) into the masseter muscle.

- Ward's or modified Ward's incision was given and mucoperiosteal flap was reflected.

- Bone guttering was done on the buccal and distal aspect stopping short of the bone on the lingual aspect.

- The tooth was sectioned using a bur, the line of sectioning being longitudinal, and the distal part was delivered out using an elevator.

- The mesial part was then luxated using a coupland's elevator and was removed next.

- The sharp edges around the socket were smoothened with a bone file.

- Bleeding was controlled and the socket was examined. The socket was irrigated with normal saline.

- 2 simple interrupted black braided silk sutures were placed.

- Pressure pack was given over the wound.

- Post-operative instructions were given to the patient.

Post-operative Medications:

- Amoxicillin+Clavulinic acid (500 mg+125 mg)-thrice daily for 5 days.

- Metronidazole (400 mg)-thrice daily for 5 days.

- Diclofenac sodium (50 mg)-thrice daily for 3 days.

The parameters of the study were to measure the pain, swelling and trismus after the injection of methylprednisolone or the placebo (normal saline) into the masseter muscle.

Pain: Pain would be measured every hour for 6 hours from the end of surgery and during the next seven days once in the morning and evening ( 8 a.m. and 8 p.m. ) using a $10 \mathrm{~cm}$ visual analog scale, the extreme scores being 'no pain' and 'worst pain imaginable. The patients would return the completed proforma on the seventh post-operative day when they are recalled for suture removal.

Swelling: Silk thread would be used to record the following distances: tragus-lip commissure, gonion-lip commissure and gonionexternal canthus of eye preoperatively, second post-operative day and seventh post-operative day.

Trismus: Maximal inter incisal opening would be measured preoperatively, second post-operative day and seventh post-operative day.

\section{Method of Statistical Analysis}

The following methods of statistical analysis have been used in this study. Data was entered in Microsoft excel and analysed using SPSS (Statistical Package for Social Science, Ver.10.0.5)

The results were averaged (mean + standard deviation) for continuous data. Normality of data was tested using Shapiro-Wilk test [6].

\section{Results}

A randomized placebo controlled triple blind study was conducted to assess the efficacy of methylprednisolone to reduce pain swelling and trismus after third molar surgery. A total of 15 patients in the age range 
Citation: Rishi D, Shetty A, Srivastava N, Rajani BC, Anshuman P, et al. (2018) Efficacy of Methylprednisolone Injected into Masseter Muscle Preoperatively in Surgical Extraction of Lower Third Molars: A Randomized Placebo Controlled Triple Blind Study. Dentistry 8: 478. doi:10.4172/2161-1122.1000478

of 19 to 34 years were included in the study. The study group includes 13 females and 2 males. The type of impaction included distoangular, mesioangular and horizontal. There were no cases of vertical impaction in our study. The ramus relationship of the impacted third molars was class I and class II. The depth of impaction was largely position A followed by position B and C. The Pederson's difficulty index scores varied from 4 to 8 . The duration of procedure varied between $20 \mathrm{~min}$ to $50 \mathrm{~min}$.

The mouth opening varied between $12 \mathrm{~mm}$ to $30 \mathrm{~mm}$ at day 2 and between $24 \mathrm{~mm}$ to $40 \mathrm{~mm}$ at day 7 . The tragus commissure measurement for swelling varied between $90 \mathrm{~mm}$ to $130 \mathrm{~mm}$ at day 2 and between $90 \mathrm{~mm}$ to $112 \mathrm{~mm}$ at day 7. The gonion commissure measurement for swelling varied between $65 \mathrm{~mm}$ and $110 \mathrm{~mm}$ at day 2 and $67 \mathrm{~mm}$ and $92 \mathrm{~mm}$ at day 7.The gonion external canthus measurement for swelling varied between $90 \mathrm{~mm}$ and $115 \mathrm{~mm}$ at day 2 and between 89 and 112 $\mathrm{mm}$ at day 7 .

The efficacy of methylprednisolone was evaluated based on its ability to reduce pain, swelling and trismus following the surgical extraction of impacted lower third molars.

- Pain: In 13 out of 15 (86.6\%) patients the methyl prednisolone group had better outcome.

- Swelling: In 12 out of $15(80 \%)$ patients the methyl prednisolone group had better outcome.

- Trismus: In 11 out of 15(73.3\%) patients the methyl prednisolone group had better outcome.

This study conclusively proves that patient comfort levels are far better with the use of methylprednisolone.

\section{Discussion}

Many authors have contributed data on the use of corticosteroids after third molar extraction. The strength of the evidence provided by a study depends on the success of its design in minimizing bias and maximizing attribution. Highest-quality scientific evidence (level 1 ++ ) [7] derives from meta-analyses, systematic reviews of randomized controlled trials (RCTs), or RCTs with a very low risk of bias and directly applicable to the target population, whereas high-quality evidence (level $1+$ ) is given by wellconducted meta-analyses, systematic reviews of RCTs, or RCTs with a low risk of bias that are directly applicable to the target population and demonstrate an overall consistency of results [8]. The aim of our study was to contribute an overall view of the use of corticosteroids in oral surgery and to update knowledge in this area (Figures 9 and 10).

In choosing an agent best suited for short-term high-dose therapy, a steroid with the minimal mineralocorticoid activity that maintains a therapeutic plasma level throughout the immediate postoperative period (when the acute inflammatory reaction is more intense) and produce minimal sodium retention should be preferred. Methylprednisolone meets these requirements, since it has no mineralocorticoid activity, the half-life is approximately 18-36 hours and the drug is 5-fold more potent than hydrocortisone [3]. The acetate forms of this drug have low solubility that acts as a sustained-release depot, giving these forms some clinical advantage when a longer effect is needed [9].

Different administration routes have been used for these drugs in oral surgery [10]. The use of oral forms might cause gastrointestinal upset, and steroids are best taken with food [11]. Effectiveness of the oral route of administration is dependent on patient compliance, and repeated dosing is required to maintain adequate blood levels during the

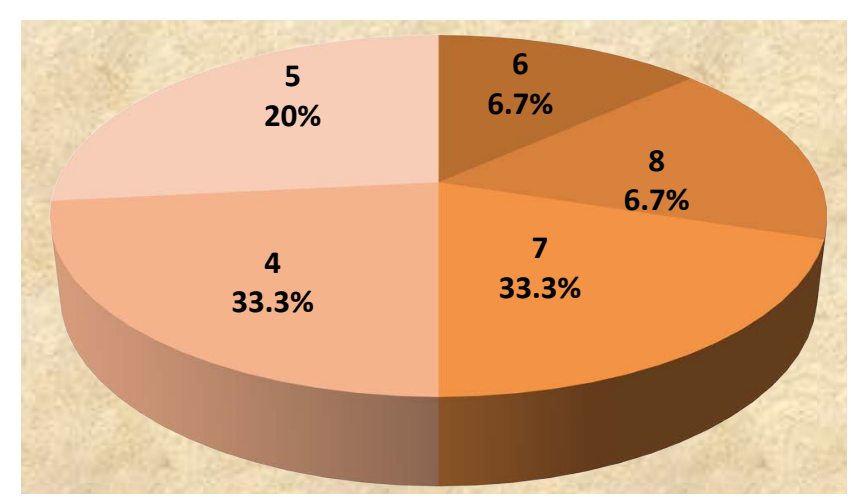

Figure 9: Distribution of Pedersons Difficulty Index among the Study Population $(\mathrm{N}=15)$.

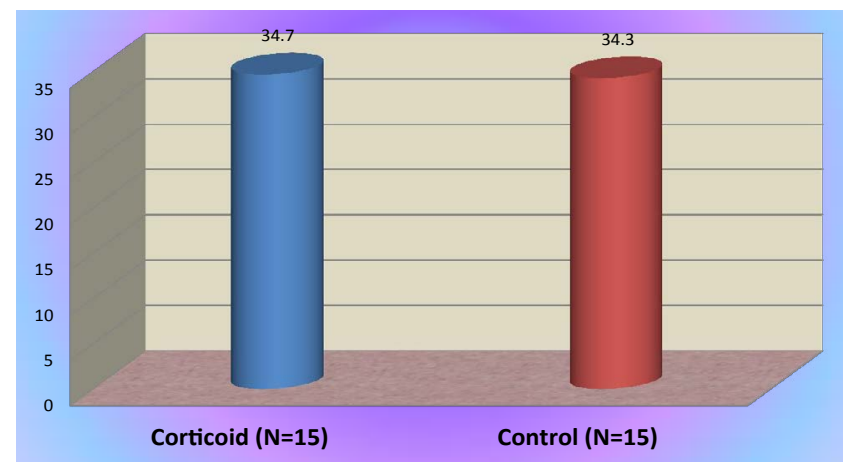

Figure 10: Comparison of Mean Duration of procedure $(\min )$ between the study groups.

postoperative period. Success of oral glucocorticosteroids in reducing the postoperative sequelae after third molar surgery is questionable [12]. The ideal route of administration is intravenous [13]. Studies using intravenous dosing suggest that a single preoperative intravenous dose results in immediate but unsustained improvement in pain, swelling, and trismus. Hence, intravenous dosing may require postoperative supplemental drug administration (oral or intramuscular) to be optimally effective [14].

One of the ways of administering the corticosteroids is in the area adjacent to the surgical site [10]. Our study evaluated the efficacy of injecting the drug in to the masseter muscle close to the surgical site.

Intramuscular administration allows the use of repository (acetate) drug forms, which provide a slow absorption and a prolonged duration of effect. Intramuscular dosing studies suggest that this route of administration can be effective in a single dose given either preoperatively or postoperatively [15]. Thus intramuscular administration of glucocorticoids alleviate the need for repeated dosing and patients' compliance.

Our study though had its own limitations. One of the limitations was although it was a bilaterally symmetrical impactions 'study the Pederson index varied between 4 to 8 . This can be improved further by choosing a specific type of impaction, e.g. class II horizontal and position B.

Another limitation was although it was a single operator study, the patients happen to be the fifteenth to seventieth lower third 
Citation: Rishi D, Shetty A, Srivastava N, Rajani BC, Anshuman P, et al. (2018) Efficacy of Methylprednisolone Injected into Masseter Muscle Preoperatively in Surgical Extraction of Lower Third Molars: A Randomized Placebo Controlled Triple Blind Study. Dentistry 8: 478. doi:10.4172/2161-1122.1000478

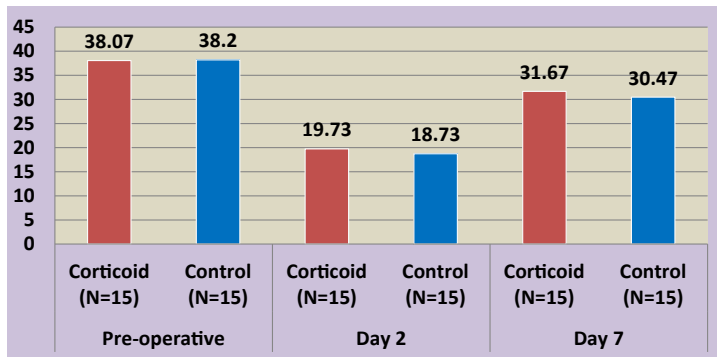

Figure 11: Comparison of Mean Oral Aperture $(\mathrm{mm})$ values between the study groups at different time points.

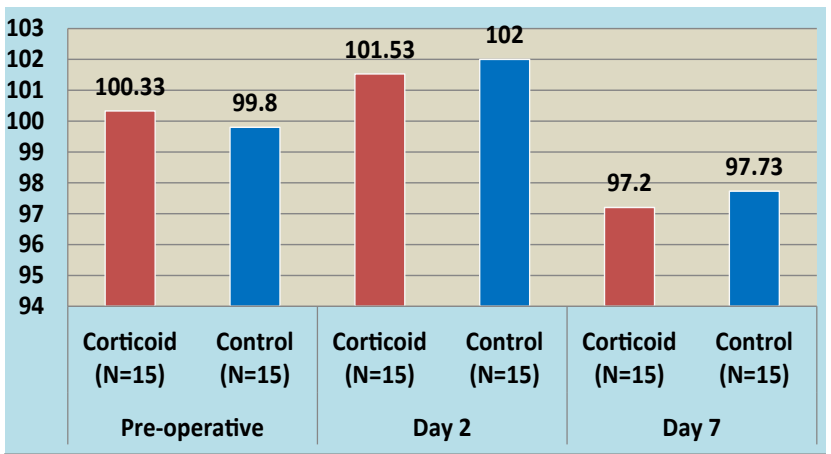

Figure 12: Comparison of Mean Tragus Commissure values between the study groups at different time points.

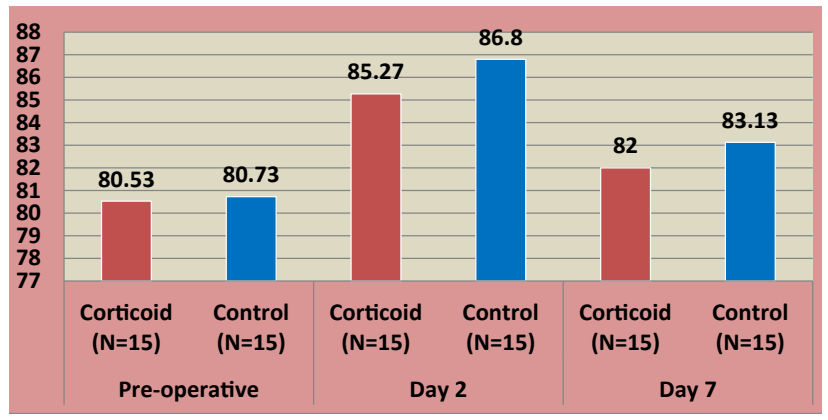

Figure 13: Comparison of Mean Gonion Commissure values between the study groups at different time points.

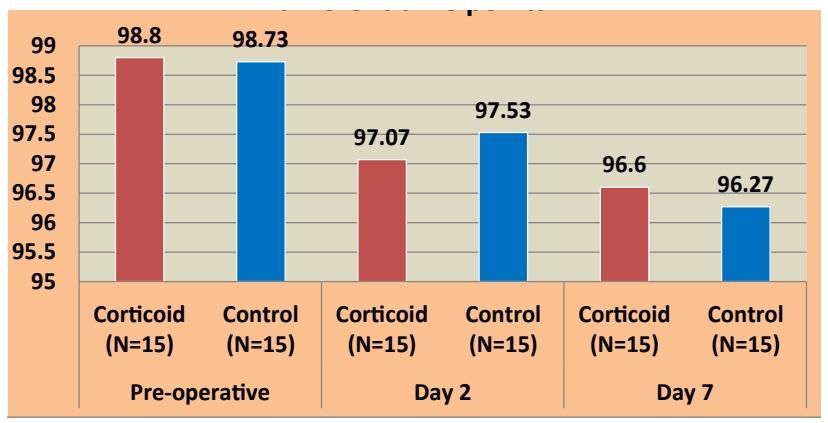

Figure 14: Comparison of Mean Gonion External Canthus values between the study groups at different time points.

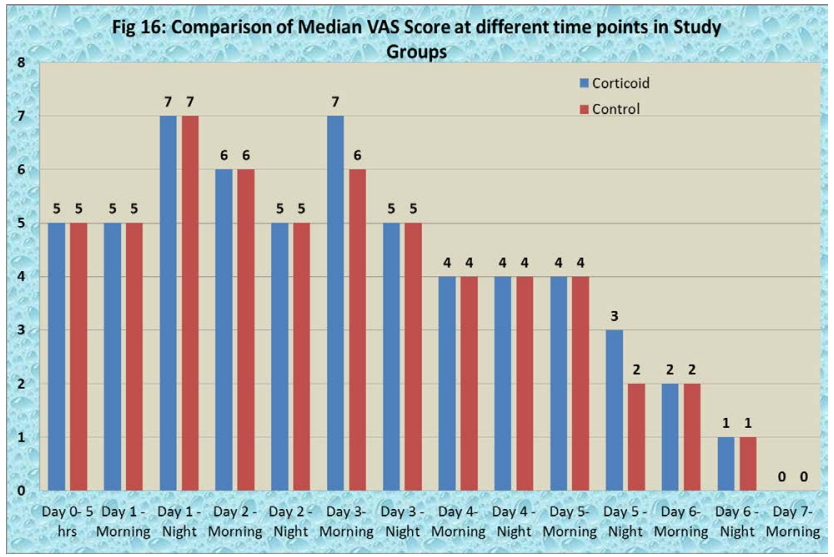

Figure 15: Comparison of Median VAS Score at different time points in Study Groups.

molar surgery case of the operator. As it is relevant in any surgery the improvement in skills is a long learning curve (Figures 11-15).

The sample size was finalized after consulting a statistician and taking into account some of the previous studies. Nevertheless higher sample size would have made the study more accurate.

\section{Conclusion}

Controlled trials are designed to overcome the weakness of observational studies. The percentage of randomized control trials in oral and maxillofacial surgery is negligible, and their reported quality is not optimal. Instead, the specialty journals host a significant number of experts' opinions.

Our study is a sincere attempt to bridge this gap. This randomized control trial has helped us to conclude that methyl prednisolone definitely has a positive outcome in terms of reducing post-operative pain, swelling and trismus after lower third molar surgery. Concerns about the safety of the drug, the time of administration and the route of administration also have been addressed in this study.

Methylprednisolone injected into masseter muscle preoperatively in surgical extraction of lower third molars can be recommended as a protocol for lower third molar surgery in particular and oral and maxillofacial surgery in general.

\section{References}

1. Bui CH, Seldin EB, Dodson T (2003) Types, frequencies, and risk factors for complications after third molar extraction. J Oral Maxillofac Surg 2003 61: 1379-1389.

2. Sisk AL, Hammer WB, Shelton DW, Joy ED (1986) Complications following removal of impacted third molars: the role of the experience of the surgeon. $J$ Oral Maxillofac Surg 44: 855-859.

3. Beirne OR, Hollander B (1986) The effect of methlyprednisolone on pain trismus, and swelling after removal of third molars. Oral Surg Oral Med Oral Pathol 61: 134-138.

4. Eeden V, Bütow K (2006) Post-operative Sequelae of Lower Third Molar Removal: A Literature Review and Pilot Study on the Effect of Covomycin. SADJ 61: 154-159.

5. Montgomery MT, Hoggs JP, Robers DL (1990) The use of glucocorticosteroids to lessen the inflammatory sequelae following third molar surgery. $\mathrm{J}$ Oral Maxillofac Surg 1990 48: 179-187.

6. www.consort-statement.org.

7. http://www.cebm.net/index.aspx?o=5653. 
Citation: Rishi D, Shetty A, Srivastava N, Rajani BC, Anshuman P, et al. (2018) Efficacy of Methylprednisolone Injected into Masseter Muscle Preoperatively in Surgical Extraction of Lower Third Molars: A Randomized Placebo Controlled Triple Blind Study. Dentistry 8: 478. doi:10.4172/2161-1122.1000478

Page 6 of 6

8. Kyzas PA (2008) Evidence-Based Oral and Maxillofacial Surgery. J Oral Maxillofac Surg 66: 973-986.

9. Milles M, Desjardins PJ (1993) Reduction of postoperative facial swelling by low-dose methylprednisolone: An experimental study. J Oral Maxillofac Surg 51: $987-991$

10. Bustumante EV, Mico-Lloens J, Gargallo-Albiol J, Satorres-Nieto, Berini-Aytes L, et al. (2008) Efficacy of methylprednisolone injected into the masseter muscle following the surgical extraction of impacted lower third molars. Int $\mathrm{J}$ Oral Maxillofac Surgery 37: 260-263.

11. Vyas N, Agarwal S, Shah N, Patel D, Aapaliya P (2014) Effect of single dose intramuscular methylprednisolone injection into the masseter muscle on the surgical extraction of impacted lower third molars: a Randomized Controlled trial. Kathmandu Univ Med J 12: 4-8.
12. Buyukkurt CM, Gungormus M, Kaya M (2006) The Effect of a Single Dose Prednisolone Withand Without Diclofenac on Pain, Trismus, and Swelling After Removal of Mandibular Third Molars. J Oral Maxillofac Surg 64: 1761-1766.

13. Kim K, Brar P, Jakubowski J, Kaltman S, Lopez E, et al. (2009) The use of corticosteroidsand nonsteroidal anti-inflammatory medication forthe management of pain and inflammationafter third molar surgery: A review of the literature. Oral Surg Oral Med Oral Pathol Oral Radiol Endod 107: 630-640.

14. Sisk AL, Bonnington GJ (1985) Evaluation of methylprednisolone and flurbiprofen for inhibition of the postoperative inflammatory response. Oral Surg Oral Med Oral Pathol 60: 137-145.

15. https://www.pfizermedicalinformation.ca/en-ca/product-monograph. 